\title{
Heritability of the limbic networks
}

\author{
Sanja Budisavljevic, ${ }^{1,2,3, \dagger}$ Jamie M. Kawadler, ${ }^{1,2, \dagger}$ Flavio Dell'Acqua, ${ }^{1,2}$ \\ Frühling V. Rijsdijk, ${ }^{4}$ Fergus Kane, ${ }^{5}$ Marco Picchioni, ${ }^{5}$ Philip McGuire, ${ }^{5}$ \\ Timothea Toulopoulou, ${ }^{5,6,7,8}$ Anna Georgiades, ${ }^{6}$ Sridevi Kalidindi, ${ }^{6}$ \\ Eugenia Kravariti, ${ }^{6}$ Robin M. Murray, ${ }^{6}$ Declan G. Murphy, ${ }^{1}$ \\ Michael C. Craig, ${ }^{1,2,9, \dagger}$ and Marco Catani ${ }^{1,2, \dagger}$
}

${ }^{1}$ Department of Forensic and Neurodevelopmental Sciences, and ${ }^{2}$ Natbrainlab, Centre for Neuroimaging Sciences, Institute of Psychiatry, Psychology and Neuroscience, King's College London, London SE5 8AF, UK, ${ }^{3}$ NEMo Laboratory, Department of General Psychology, University of Padova, 35131 Padova, Italy, ${ }^{4}$ Social, Genetic and Developmental Psychiatry Research Centre, ${ }^{5}$ Department of Psychological Medicine, and ${ }^{6}$ Department of Psychosis Studies, Institute of Psychiatry, Psychology and Neuroscience, King's College London, London SE5 8AF, UK, ${ }^{7}$ Department of Psychology, and ${ }^{8}$ State Key Laboratory of Brain and Cognitive Sciences, The University of Hong Kong, Hong Kong, Hong Kong, and ${ }^{9}$ National Autism Unit, South London and Maudsley NHS Foundation Trust, Beckenham, UK.

Correspondence should be addressed to Sanja Budisavljevic and Marco Catani, Natbrainlab, Section of Brain Maturation PO50, Department of Forensic and Neurodevelopmental Sciences, King's College London, Institute of Psychiatry, Psychology and Neuroscience, De Crespigny Park, London SE5 8AF, UK. E-mail: sanja.budisavljevic@gmail.com, m.catani@iop.kcl.ac.uk

${ }^{\dagger}$ These authors contributed equally to this article.

\begin{abstract}
Individual differences in cognitive ability and social behaviour are influenced by the variability in the structure and function of the limbic system. A strong heritability of the limbic cortex has been previously reported, but little is known about how genetic factors influence specific limbic networks. We used diffusion tensor imaging tractography to investigate heritability of different limbic tracts in 52 monozygotic and 34 dizygotic healthy adult twins. We explored the connections that contribute to the activity of three distinct functional limbic networks, namely the dorsal cingulum ('medial default-mode network'), the ventral cingulum and the fornix ('hippocampal-diencephalic-retrosplenial network') and the uncinate fasciculus ('temporo-amygdala-orbitofrontal network'). Genetic and environmental variances were mapped for multiple tract-specific measures that reflect different aspects of the underlying anatomy. We report the highest heritability for the uncinate fasciculus, a tract that underpins emotion processing, semantic cognition, and social behaviour. High to moderate genetic and shared environmental effects were found for pathways important for social behaviour and memory, for example, fornix, dorsal and ventral cingulum. These findings indicate that within the limbic system inheritance of specific traits may rely on the anatomy of distinct networks and is higher for fronto-temporal pathways dedicated to complex social behaviour and emotional processing.
\end{abstract}

Key words: heritability; limbic system; cingulum; uncinate fasciculus; fornix; diffusion tractography 


\section{Introduction}

Socio-emotional functioning and behaviour depend on the coordinated activity of the limbic system, composed of specific cortical and subcortical regions interconnected by white matter tracts (Catani et al., 2012). These tracts are implicated in a variety of neuropsychiatric disorders when deficits in emotion regulation, social behaviour and memory are present (Kubicki et al., 2003; Mori et al., 2007; Metzler-Baddeley et al., 2011, 2012; Nortje et al., 2013; Douet and Chang, 2014; Ameis and Catani, 2015; Whitford et al., 2015).

Recently, our group has proposed a revised model of the limbic system, with limbic tracts connecting three distinct, yet partially overlapping functional networks (Catani et al., 2013a). The first functional network, the 'dorsomedial default-mode network', consists of a group of medial regions interconnected by dorsal cingulum, and is involved in mental activities associated with the 'resting state' such as introspection, social, moral and affective processing. The dorsal cingulum is the uppermost component of the cingulum bundle, and runs along the length of the cingulate gyrus. Altered activations of the default-mode network are observed in various neuropsychiatric disorders (Broyd et al., 2009), including schizophrenia (Koch et al., 2013), psychopathy (Sethi et al., 2015), depression (Sheline et al., 2009), and so on. The second network, the 'hippocampal-diencephalic-retrosplenial' network, encompasses the fornix and ventral cingulum, which are dedicated to memory and spatial orientation. The fornix is a small projection tract with a commissural component, which links hippocampus with the mammilary body, the anterior thalamic nuclei and the hypothalamus (Aggleton, 2008). The ventral cingulum is the posterior ventral component of the cingulum bundle running within the parahippocampal gyrus and retrosplenial cingulate gyrus (Catani et al., 2013a). Lesions of this network are associated with memory (Valenstein et al., 1987) and spatial orientation deficits (Vann et al., 2009) and are implicated in neurodegenerative disorders, such as Alzheimer's disease (Buckner et al., 2005), temporal lobe epilepsy (Concha et al., 2010), mild cognitive impairment (Nestor et al., 2003), and schizophrenia (Fitzsimmons et al., 2014). The third functional network, the 'temporo-amygdala-orbitofrontal network', is involved in the integration of visceral and emotional states with cognition and behaviour (Mesulam, 2000). Its main tract is the uncinate fasciculus that runs from the anterior part of the temporal lobe, parahippocampal gyrus, uncus and amygdala to the orbital and polar frontal cortex (Catani et al., 2002). The uncinate fasciculus has been linked to different aspects of social development (Elison et al., 2013), recognition of complex emotions (Fujie et al., 2008), semantic cognition (Catani et al., 2013a,b; Catani and Mesulam, 2008; Catani and Bambini, 2014), and social behavior (D'Anna et al., in press). Damage to this network is related to deficits in socio-emotional processing in disorders such as autism (Pugliese et al., 2009; Kumar et al., 2010; Thomas et al., 2011; Catani et al., in press), psychopathy (Craig et al., 2009), borderline personality disorder (Lischke et al., 2015), apathy (Hollocks et al., 2015), mood disorders (McIntosh et al., 2008; Carballedo et al., 2012; Zhang et al., 2012), obsessive-compulsive disorder (Peng et al., 2014), anorexia nervosa (Lipsman et al., 2015), and alcoholism (Schulte et al., 2012). Overall, individual differences in socio-emotional behaviour and vulnerability to specific neuropsychiatric disorders have been associated with the variability in the structure of the three limbic networks listed above.

Hence, understanding the relative influence of genetic and environmental factors on the structure of limbic tracts can help to clarify the biological mechanisms underlying predisposition to vulnerability and resilience to neuropsychiatric disorders. The classical twin design provides a unique method for establishing heritability by comparing monozygotic (MZ) and dizygotic (DZ) twins raised in the same family. The heritability is reflected in the difference in correlations between $\mathrm{MZ}$ and $\mathrm{DZ}$ twins, because MZ twins share all their genetic sequence while DZ twins share approximately $50 \%$ of their genes. If $\mathrm{MZ}$ twins resemble each other more than DZ twins, the trait can be assumed to be heritable. Furthermore, using structural equation modelling (SEM) the phenotypic variance can be parsed into genetic and environmental components (shared environmental and specific environmental factors, which include the measurement error) (Plomin et al., 2013).

Using twin design, limbic system functions such as social cognition (Scourfield et al., 1999), associative memory (Thapar et al., 1994), aggressive behaviour (Craig and Halton, 2009), prosocial behaviour and empathy (Matthews et al., 1981; Rushton et al., 1984, 1986; Knafo and Israel, 2009), antisocial behaviour (Viding et al., 2005), parenting (Losoya et al., 1997), anxietyrelated personality characteristics (Stein et al., 2002) and personality measures such as psychoticism, extraversion, and neuroticism (Plomin et al., 1994; Eaves et al., 1999) are found to be moderately to highly heritable throughout lifespan. However, certain traits are more heritable than others, for example, the personality traits (46-51\%) and spatial reasoning (40\%) compared to memory (22\%) (Plomin et al., 1994). Moreover, heritability seems to be age dependent, and the twin literature on prosocial traits, empathy, externalizing behaviours, but also anxiety and depressive symptoms, indicates the increasing importance of genes with increasing age, while shared environmental effects decrease in importance from early childhood to adulthood (Bergen et al., 2007; Ebstein et al., 2010).

Structural brain imaging studies in healthy twins report moderate to high heritability for limbic grey matter regions such as posterior cingulate gyrus (51-75\%), hippocampus (64-71\%), and amygdala (55-71\%) (Wright et al., 2002; Hulshoff Pol et al., 2006; Kremen et al., 2010). Hence, it is not surprising that limbic structures act as putative endophenotypes in different disorders such as schizophrenia (Weinberger et al., 1992; McDonald et al., 2004; Rijsdijk et al., 2005; van Haren et al., 2012; Roalf et al., 2015), bipolar disorder (McDonald et al., 2004), epilepsy (Scanlon et al., 2013; Alhusaini et al., 2015), and dementia (Järvenpää et al., 2004).

Little is known about genetic and environmental influences on specific limbic white matter tracts. Advances in diffusion tensor imaging (DTI) tractography permit to reliably reconstruct and study specific white matter pathways in vivo. To date, only three studies have used DTI tractography to explore genetics of limbic pathways in childhood. One study on neonates (Lee et al., 2015) reported high heritability of the anatomy of fornix and ventral cingulum (hippocampal-diencephalic-retrosplenial network), compared to the uncinate fasciculus and dorsal cingulum (temporo-amygdala-orbitofrontal and medial default-mode networks) that are known to mature later in life (Lebel et al., 2008). With increasing age from early to late childhood, a genetic effect seems to significantly decrease for the fornix, while it moderately increases for the uncinate and more strongly for the cingulum (Brouwer et al., 2010, 2012). Previous DTI studies on adults have been conducted using voxel-based methods that lack the ability to answer questions regarding specific fibre tracts. Nevertheless, these studies suggest low heritability for the microstructure of fornix (Jahanshad et al., 2013), moderate to high for cingulum (Kochunov et al., 2010; Gatt et al., 2012; Jahanshad et al., 2013; Shen et al., 2014), and high for uncinate fasciculus (Gatt et al., 2012). Overall, studies indicate that heritability of limbic pathways is heterogeneous and changes with increasing age (Lenroot et al., 2009). 
To the best of our knowledge, there is no DTI study that has explored heritability of limbic tracts in healthy adults using tractography. Here we used DTI tractography and univariate twin modelling to study the heritability of specific limbic pathways in healthy adults. We compared the dorsal cingulum (medial default-mode network), the ventral cingulum and the fornix (hippocampal-diencephalic-retrosplenial network) and the uncinate fasciculus (temporo-amygdala-orbitofrontal network) in $52 \mathrm{MZ}$ and $34 \mathrm{DZ}$ adult twins. The contributions of genes and environment were studied on multiple tract-specific measures including volume, fractional anisotropy and mean diffusivity that reflect different aspects of the underlying anatomy, microarchitecture, and biology.

\section{Methods \\ Participants}

In this study, we included 86 healthy right-handed adult twins, $26 \mathrm{MZ}$ and $17 \mathrm{DZ}$ twin pairs, recruited from a volunteer twin register at the Institute of Psychiatry, Psychology and Neuroscience (London, UK) and by national media advertisements. Mean age for MZ twins was 35.5 years (age range 21-56 years), and 42.5 years (age range 20-62 years) for DZ twins. All DZ twin pairs included in this study were same-sex pairs. Opposite-sex pairs were excluded to avoid inflation of heritability estimates due to an overall lower DZ correlation due to qualitative sex differences (Stromswold, 2001). Of the $26 \mathrm{MZ}$ twin pairs, 9 pairs were male and 17 female. Of the $17 \mathrm{DZ}$ twin pairs, 4 pairs were male and 13 were female. Other exclusion criteria included a history of neurological disorder or systemic illness with known neurological complications, significant head injury associated with loss of consciousness for more than one minute and current harmful substance use or dependence (defined as within the last 12 months). A UK Multicentre Research Ethics Committee approved the study, and all the participants gave written informed consent before participating. Due to movement and scanner artefacts we excluded two DZ twin pairs from the cingulum analysis, four DZ twin pairs from the uncinate fasciculus analysis, and two MZ twin pairs and one $\mathrm{DZ}$ twin pair from the fornix analysis.

\section{Data acquisition and tractography analysis}

DTI data were acquired using a GE Signa 1.5-T LX MRI system (General Electric, Milwaukee, WI) with maximum gradient amplitude of $40 \mathrm{mT} / \mathrm{m}$ and an acquisition sequence fully optimized for DTI and tractography of white matter. All images were acquired from the whole brain, with sections parallel to the anterior-posterior commissure line. Using a peripherally gated echo planar imaging pulse sequence, each DTI-MRI volume was acquired from 60 contiguous $2.5 \mathrm{~mm}$ thick slices with a field of view of $240 \times 240 \mathrm{~mm}$ and an acquisition matrix size of $96 \times 96$ pixels, giving an isotropic voxel size of $2.5 \mathrm{~mm} \times 2.5 \mathrm{~mm} \times 2.5 \mathrm{~mm}$. Echo time was $102 \mathrm{~ms}$. Acquisitions were peripherally gated with an effective repetition time of $15 \mathrm{R}-\mathrm{R}$ intervals. At each location, 7 images were acquired without diffusion weighting, together with 64 images with a weighting of $1300 \mathrm{~s} / \mathrm{mm}^{2}$ applied along directions uniformly distributed in space.

Diffusion data were analysed using ExploreDTI (Leemans and Jones, 2009). Following correction for eddy current distortions and subject motion, the diffusion tensor (DT) was estimated using a non-linear least square methods and fractional anisotropy (FA) and mean diffusivity (MD) maps were estimated. Whole brain tractography was performed using a Euler integration to propagate streamlines following the directions of the principal eigenvector with a step size of $0.5 \mathrm{~mm}$. Tractography was started in all brain voxels with $F A>0.2$. Streamlines were tracked until the FA of the tensor was above an FA threshold of 0.2 or the curvature (i.e. the angle between two consecutive steps) was less than $30^{\circ}$.

\section{Virtual dissections of the limbic system pathways}

The tractography data were visualized using TrackVis (Massachusetts General Hospital, Boston, MA) to perform the virtual dissection of white matter bundles.

Uncinate fasciculus. We used a two-regions of interest (ROI) approach to dissect the uncinate fasciculus, in line with the method previously described by our laboratory (Catani and Mesulam, 2008). The first ROI was placed in the white matter of the anterior floor of the external/extreme capsule on contiguous axial slices, while the second ROI was placed in the white matter of the anterior temporal lobe.

Fornix. We used a one-ROI approach to dissect the fornix, as described previously by our laboratory (Catani and Thiebaut de Schotten 2008). This choice was made because the fornix contains a commissural component known as the hippocampal commissure, and thus we did not separate the left and the right columns. Thus, a single ROI is placed around the body of the fornix on contiguous coronal slices inferior to the corpus callosum. To better visualize the entire course of the fornix into the temporal lobe, additional regions around the fimbriae of the left and right side were included in the ROI.

Dorsal and ventral cingulum. In this study, we introduce a novel method for segmenting the cingulum bundle into its dorsal and ventral components, in contrast to the one-ROI approach for dissecting the whole cingulum bundle described previously by our group (Catani and Thiebaut de Schotten 2008). Previous studies have attempted segmentation based on a single ROI approach (Sun et al., 2003; Wu et al., 2010), a two-ROI approach investigating either the long fibres of the dorsal cingulum or ventral cingulum (Zhang et al., 2007; Nakata et al., 2009; Takei et al., 2009; Kamagata et al., 2012), or dissection of the cingulum based on multiple ROIs placed at specific points along the fasciculus (Catani et al., 2002; Kim et al., 2006; Catheline et al., 2010). Importantly, the cingulum bundle consists of fibres of different length including the long fibres and the short U-shaped fibres. For that reason, we used an updated one-ROI approach from Catani and Thiebaut de Schotten (2008), which identifies both types of cingulum fibers, unlike other two-ROI approaches that exclude the majority of the short U-shaped fibres from the analysis. Thus, we used a one-ROI approach separately for both dorsal and ventral cingulum as following. First, a vertical midline bisecting the splenium of the corpus callosum was delineated on a midsagittal coronal slice. For the dissection of the dorsal cingulum, a single ROI was placed on contiguous coronal slices around the cingulum bundle anterior to the vertical midline of the splenium and superior to the body of the corpus callosum. For the ventral cingulum, a single ROI was delineated on contiguous slices posterior to the vertical midline of the splenium and inferiorly along the medial temporal lobe/parahippocampal gyrus (Figure 1). Thus, besides distinguishing dorsal and ventral cingulum, our novel approach allowed for visualization not only of the long association fibres, but also of the U-shaped local 
fibres that connect the neighbouring gyri and represent the majority of the cingulum fibres.

\section{Statistical analysis}

For each limbic tract, namely fornix and bilateral dorsal cingulum, ventral cingulum, and uncinate fasciculus, the number of voxels touched by the tract streamlines was calculated as a surrogate measure of the tract volume (referred from now on as Volume), together with average tract-specific measures of FA and $\mathrm{MD}$. We confirmed that these quantitative measures were normally distributed, and that there was homogeneity of means and variances across the measures (using paired t-tests and Levene's test, respectively).

A comparison of intra-class correlation coefficients (ICCs) of DTI measures in MZ and DZ twins provided initial descriptive statistics on the presence of genetic effects. An MZ ICC that is at least twice as large as a DZ ICC indicates that this measure is largely influenced by genetic factors. An MZ correlation larger than, but less than twice the DZ correlation, indicates the

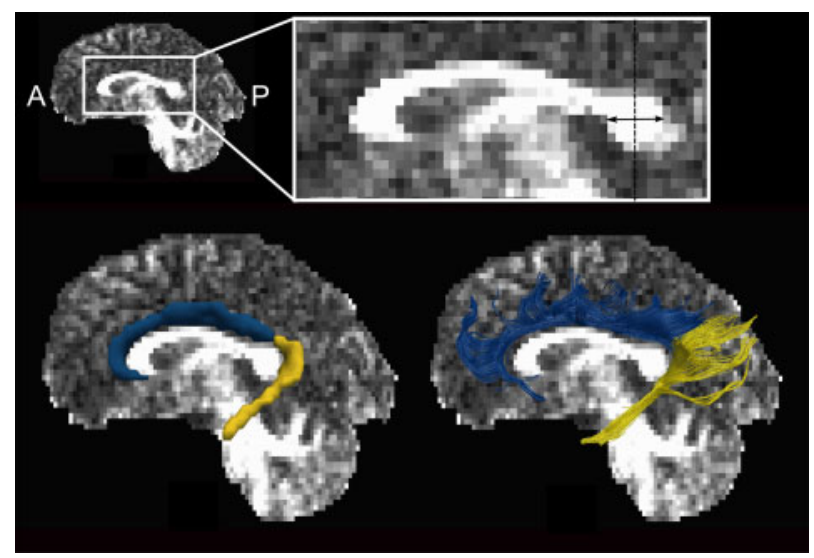

Fig. 1. Dissected dorsal (blue) and ventral (yellow) components of the cingulum bundle. The vertical midline of the splenium of the corpus callosum separates the uppermost anterior coronal slices of the dorsal cingulum from the lowermost slices of the ventral cingulum. When the width of the splenium is an even number of voxels and cannot be halved easily, the consistent method is to select the first voxel of the posterior half of the splenium. This slice is included in the dorsal cingulum ROI (blue). significant effect of shared environmental factors (all non-genetic factors that make family members more alike).

Prior to quantitative genetic model fitting of the extracted diffusion measures from the limbic connections, age, sex and handedness were regressed out using SPSS, and residuals standardized for subsequent analysis. On that note, we have recently reported the absence of gender differences among the limbic tracts studied suggesting that this bias was unlikely to influence the findings (Thiebaut de Schotten et al., 2011). Model fitting was carried out using SEM program OpenMx (Boker et al., 2011, 2012), to provide heritability parameter estimates and their confidence intervals (Neale and Maes, 2011). Sources of variance in the DTI-extracted measures were divided into additive genetic (A) effects, shared environmental $(C)$ effects, environmental influences that make the twins more similar, and non-shared or specific environmental influences $(E)$ that contribute to differences between the twins and also include a possible measurement error (Plomin and Kosslyn, 2001). The proportion of variability that can be attributed to additive genetic factors is called 'heritability'. Maximum likelihood estimates of $A, C$ and $E$ were obtained (values range from 0 to 1 , where $A+C+E=1$ ), with their $95 \%$ confidence intervals calculated and a series of nested models compared. A full ACE model was compared to the nested $\mathrm{AE}, \mathrm{CE}$ and $\mathrm{E}$ models (testing the effects of common environmental factors, additive genetic factors and all familial resemblance, respectively). However, in cases where effect sizes, sample sizes or trait prevalence are low, statistical power for univariate twin analyses can become an issue (Neale et al., 1994) and thus only full ACE model estimates are reported. We did not specifically model familial factors, but we analysed E-only models to test for familial influences which refer to a significant combined effect of A + C. Goodness-of-fit probability for the ACE model $>0.05$ suggests a good fit (the opposite of the usual $P$-value convention). Estimates are significant if the reported maximum likelihood $95 \%$ confidence intervals do not contain zero.

\section{Results}

\section{Intra-class correlation coefficients}

The ICC results for FA, MD and volume measures extracted from four limbic tracts are shown in Tables 1-4. Overall, the ICCs for the MZ twins were either higher or similar to those of

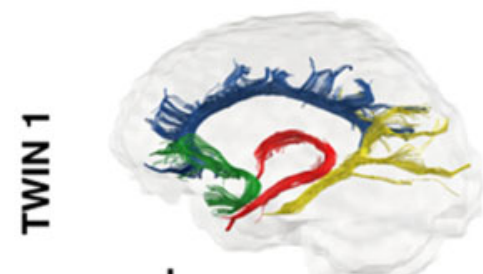

L

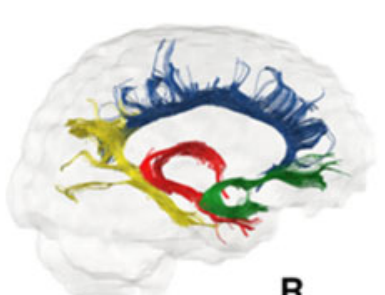

MZ

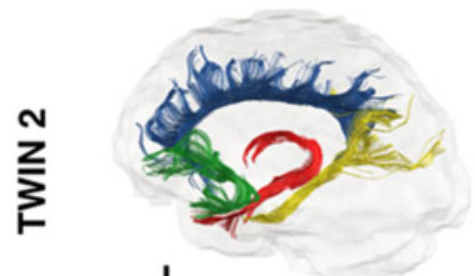

L

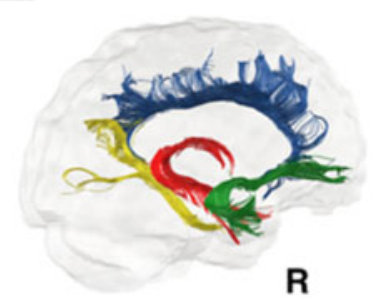

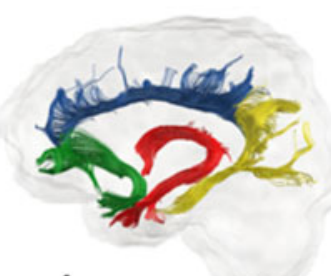

L

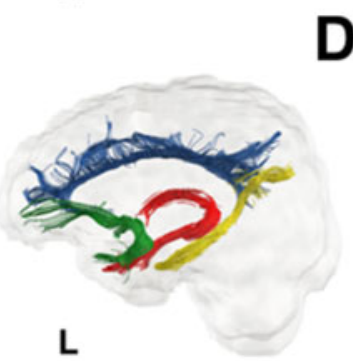

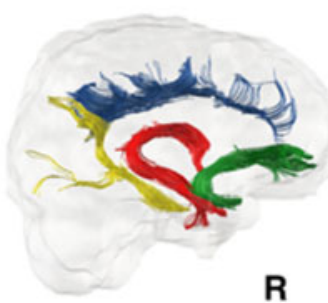

$\mathbf{R}$
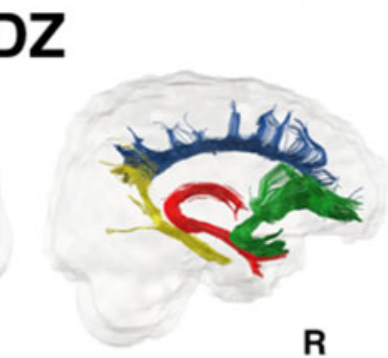

Fig. 2. Representative examples of the four limbic tracts dissected in MZ and DZ twin pairs. The dorsal cingulum is shown in blue, the ventral cingulum in yellow, the fornix in red and the uncinate fasciculus in green colour. 
DORSAL CINGULUM

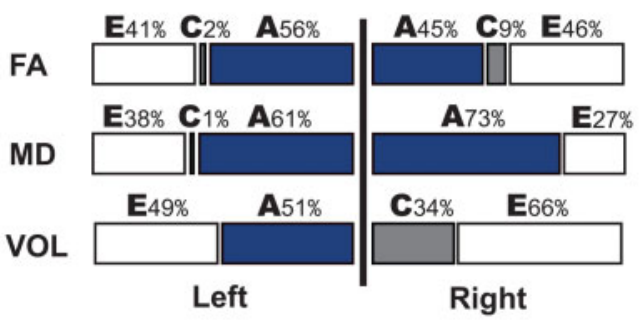

VENTRAL CINGULUM

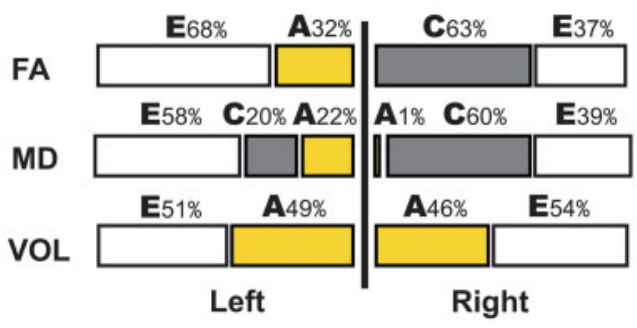

UNCINATE FASCICULUS

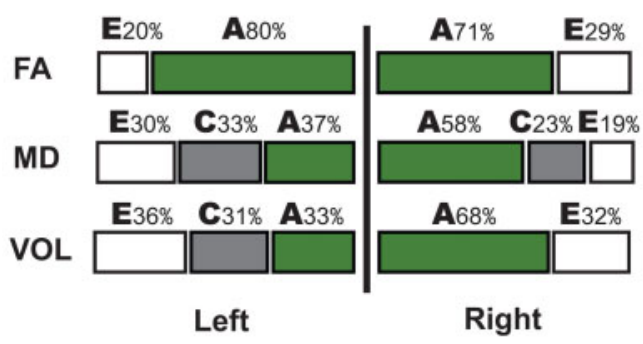

\section{FORNIX}

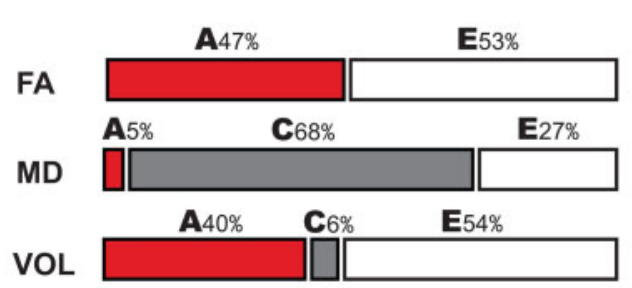

Fig. 3. The relative contribution (in percentages) of genetic (A), shared environmental (C) and specific environmental (E) factors on the variability of the FA, MD and volume of fornix (red), the bilateral dorsal (blue) and ventral (yellow) cingulum, and the bilateral uncinate fasciculus (green)

the DZ twins. In MZ twins, these correlations were statistically significant in all limbic tracts $(P<0.05)$. This may suggest high genetic or shared environmental effects on the anatomy of the limbic tracts. The ICCs of the right MD in both ventral cingulum and fornix were high and significant in both MZ and DZ twins, suggesting presence of shared environmental effects. Representative examples of the anatomy of the four limbic tracts in MZ and DZ twin pairs are shown in Figure 2. Genetic and environmental effects were formally tested using SEM.

\section{Structural equation modelling}

SEM was used to extract the relative contributions of additive genetic $(A)$, common environmental $(C)$ and unique environmental (E) factors on the variability of the volume, FA and MD measures of the four limbic tracts (Tables 1-4). The width of the confidence intervals was sometimes considerable due to the relatively small sample size of our study compared to the standard behavioural genetics studies. This meant that for some measures, the individual genetic (A) and shared environmental (C) effects were non-significant, but significant when tested together as familial effect $(A+C)$ (Wright et al., 2002). The $P$ values shown in Tables 1-4 indicate the fit of the ACE model compared to the saturated model; values above 0.05 indicate a good fit of the model. The results are presented according to the threenetwork model of the limbic system (Figure 3).

Heritability of dorsomedial 'default' network. Dorsal cingulum. Specific environmental factors ( $E$ ) accounted for $49 \%$ and $66 \%$ of variance in the left and right volume of dorsal cingulum, respectively, with significant familial effect present only in the left hemisphere $(51 \%)$, and smaller insignificant familial effect $(36 \%)$ in the right hemisphere where the E model was the best fit. In contrast to the volume, genetics $(A)$ accounted for most of the variance in the right MD (73\%), while significant familial effects $(A+C)$ accounted for $62 \%$ of left MD. Heritability of FA was high and accounted for $56 \%$ and $45 \%$ of variance in the left and right hemisphere respectively (Table 1).

Heritability of hippocampal-diencephalic retrosplenial network. Ventral cingulum. Specific environmental factors (E) significantly explained most of the variance in FA and MD of the left ventral cingulum (68\% and $58 \%$, respectively). In contrast, the variability in FA and MD in the right hemisphere was influenced mostly by the familial factors $(A+C)(61-63 \%)$, and less so by specific 
Table 1. dCG MZ and DZ ICC coefficients of FA, MD and volume in the left and right hemisphere, with standardized additive genetic (A), common environmental $(C)$ and unique environmental $(E)$ variance components of the full ACE model (and 95\% CI) with the associated DF, chisquare and $P$ values

\begin{tabular}{|c|c|c|c|c|c|c|c|c|}
\hline dCG & ICC (MZ) & ICC (DZ) & A & C & E & Best fit model* & $\chi^{2}(\mathrm{DF}=6)$ & $P$ value \\
\hline FA (L) & $0.63^{* *}(0.32-0.81)$ & $0.29(-0.25$ to 0.80$)$ & $0.56(0-0.77)$ & $0.02(0-0.67)$ & $0.42(0.23-0.70)$ & $(A+C) E$ & 1.62 & 0.95 \\
\hline $\mathrm{FA}(\mathrm{R})$ & $0.52^{* *}(0.18-0.75)$ & $0.39(-0.12$ to 0.74$)$ & $0.45(0-0.75)$ & $0.09(0-0.63)$ & $0.46(0.25-0.80)$ & $(A+C) E$ & 1.52 & 0.96 \\
\hline $\mathrm{MD}(\mathrm{L})$ & $0.53^{* *}(0.19-0.76)$ & $0.42^{*}(-0.09$ to 0.75$)$ & $0.61(0-0.80)$ & $0.01(0-0.60)$ & $0.38(0.20-0.74)$ & $(A+C) E$ & 4.76 & 0.58 \\
\hline $\mathrm{MD}(\mathrm{R})$ & $0.76^{* *}(0.53-0.88)$ & $0.29(-0.23$ to 0.68$)$ & $0.73(0.10-0.86)$ & $0(0-0)$ & $0.27(0.14-0.49)$ & $A E$ & 4.35 & 0.63 \\
\hline $\operatorname{Vol}(\mathrm{L})$ & $0.60^{* *}(0.29-0.80)$ & $0.03(-0.46$ to 0.51$)$ & $0.51(0-0.71)$ & $0(0-0.65)$ & $0.49(0.29-0.77)$ & $(A+C) E$ & 7.81 & 0.25 \\
\hline Vol (R) & $0.36^{*}(-0.02$ to 0.65$)$ & $0.35(-0.16$ to 0.72$)$ & $0(0-0.56)$ & $0.34(0-0.58)$ & $0.66(0.42-0.96)$ & $E$ & 4.08 & 0.67 \\
\hline
\end{tabular}

dCG, dorsal cingulum; L, left; $R$, right.

${ }^{*} \mathrm{P}<0.05,{ }^{* *} \mathrm{P}<0.01$, ICC test.

Table 2. vCG MZ and DZ ICC coefficients of FA, MD and volume in the left and right hemisphere, with standardized additive genetic (A), common environmental $(C)$ and unique environmental $(E)$ variance components of the full ACE model (and 95\% CI) with the associated DF, chisquare and $P$ values

\begin{tabular}{|c|c|c|c|c|c|c|c|c|}
\hline vCG & ICC (MZ) & ICC (DZ) & A & C & E & Best fit model ${ }^{*}$ & $\chi^{2}(\mathrm{DF}=6)$ & $P$ value \\
\hline FA (L) & $0.35^{*}(-0.03$ to 0.65$)$ & $0.15(-0.36$ to 0.60$)$ & $0.32(0-0.59)$ & $0(0-0)$ & $0.68(0.41-1)$ & E & 1.62 & 0.95 \\
\hline $\mathrm{FA}(\mathrm{R})$ & $0.66^{* *}(0.37-0.84)$ & $0.59^{*}(0.10-0.85)$ & $0(0-0.74)$ & $0.63(0-0.79)$ & $0.37(0.21-0.61)$ & $(A+C) E$ & 5.25 & 0.51 \\
\hline $\mathrm{MD}(\mathrm{L})$ & $0.42 *(0.05-0.69)$ & $0.37(-0.14$ to 0.73$)$ & $0.22(0-0.68)$ & $0.20(0-0.60)$ & $0.58(0.32-0.91)$ & $(A+C) E$ & 5.29 & 0.51 \\
\hline $\mathrm{MD}(\mathrm{R})$ & $0.63^{* *}(0.33-0.81)$ & $0.62^{* *}(0.20-0.85)$ & $0.01(0-0.74)$ & $0.60(0-0.77)$ & $0.39(0.21-0.62)$ & $(A+C) E$ & 3.41 & 0.76 \\
\hline Vol (L) & $0.57^{* *}(0.24-0.78)$ & $0.01(-0.49$ to 0.52$)$ & $0.49(0-0.71)$ & $0(0-0.59)$ & $0.51(0.30-0.82)$ & $(A+C) E$ & 4.50 & 0.61 \\
\hline $\mathrm{Vol}(\mathrm{R})$ & $0.53^{* *}(0.17-0.77)$ & $0.05(-0.45$ to 0.53$)$ & $0.46(0-0.70)$ & $0(0-0.55)$ & $0.54(0.31-0.88)$ & $(A+C) E$ & 4.35 & 0.63 \\
\hline
\end{tabular}

vCG, ventral cingulum; L, left; R, right.

${ }^{*} \mathrm{P}<0.05,{ }^{* *} \mathrm{P}<0.01$, ICC test.

Table 3. Fx MZ and DZ ICC coefficients of FA, MD and volume, with standardized additive genetic (A), common environmental (C) and unique environmental $(E)$ variance components of the full ACE model (and $95 \% \mathrm{CI}$ ) with the associated DF, chi-square and $P$ values

\begin{tabular}{|c|c|c|c|c|c|c|c|c|}
\hline $\mathrm{Fx}$ & ICC (MZ) & ICC (DZ) & A & C & $E$ & Best fit model ${ }^{*}$ & $\chi^{2}(\mathrm{DF}=6)$ & $P$ value \\
\hline FA & $0.52^{* *}(0.16-0.76)$ & $0.06(-0.44$ to 0.54$)$ & $0.47(0-0.72)$ & $0(0-0.47)$ & $0.53(0.27-0.90)$ & $(A+C) E$ & 3.59 & 0.73 \\
\hline $\mathrm{MD}$ & $0.74^{* *}(0.45-0.89)$ & $0.72^{* *}(0.36-0.89)$ & $0.05(0-0.78)$ & $0.68(0-0.85)$ & $0.27(0.13-0.49)$ & $(A+C) E$ & 6.50 & 0.37 \\
\hline Vol & $0.48^{* *}(0.10-0.73)$ & $0.29(-0.21$ to 0.67$)$ & $0.40(0-0.70)$ & $0.06(0-0.60)$ & $0.54(0.30-0.89)$ & $(A+C) E$ & 3.68 & 0.72 \\
\hline
\end{tabular}

Fx, fornix.

${ }^{*} \mathrm{P}<0.05,{ }^{* *} \mathrm{P}<0.01$, ICC test.

Table 4. UF MZ and DZ ICC coefficients of FA, MD and volume in the left and right hemisphere, with standardized additive genetic (A), common environmental $(C)$ and unique environmental $(E)$ variance components of the full ACE model (and 95\% CI) with the associated DF, chi-square and $P$ values

\begin{tabular}{|c|c|c|c|c|c|c|c|c|}
\hline UF & ICC (MZ) & ICC (DZ) & A & C & $E$ & Best fit model ${ }^{*}$ & $\chi^{2}(\mathrm{DF}=6)$ & $P$ value \\
\hline FA (L) & $0.78^{* *}(0.57-0.89)$ & $0.34(-0.21$ to 0.74$)$ & $0.80(0.29-0.89)$ & $0(0-0.46)$ & $0.20(0.10-0.40)$ & $A E$ & 1.16 & 0.98 \\
\hline FA (R) & $0.66^{* *}(0.38-0.83)$ & $0.43(-0.11$ to 0.78$)$ & $0.71(0-0.84)$ & $0(0-0.61)$ & $0.29(0.15-0.57)$ & $(A+C) E$ & 2.22 & 0.90 \\
\hline $\mathrm{MD}(\mathrm{L})$ & $0.69^{* *}(0.43-0.85)$ & $0.57^{*}(0.07-0.84)$ & $0.37(0-0.83)$ & $0.33(0-0.76)$ & $0.30(0.16-0.55)$ & $(A+C) E$ & 0.66 & 1.00 \\
\hline $\mathrm{MD}(\mathrm{R})$ & $0.80^{* *}(0.61-0.90)$ & $0.58^{*}(0.10-0.85)$ & $0.58(0.03-0.90)$ & $0.23(0-0.72)$ & $0.19(0.09-0.37)$ & $\mathrm{AE}$ & 3.89 & 0.69 \\
\hline $\operatorname{Vol}(\mathrm{L})$ & $0.64^{* *}(0.35-0.82)$ & $0.54^{*}(0.03-0.83)$ & $0.33(0-0.80)$ & $0.31(0-0.74)$ & $0.36(0.19-0.64)$ & $(A+C) E$ & 0.26 & 1.00 \\
\hline Vol (R) & $0.63^{* *}(0.33-0.81)$ & $0.43(-0.11$ to 0.78$)$ & $0.68(0-0.83)$ & $0(0-0.62)$ & $0.32(0.17-0.62)$ & $(A+C) E$ & 5.80 & 0.45 \\
\hline
\end{tabular}

UF, uncinate fasciculus; L, left; R, right.

${ }^{*} \mathrm{P}<0.05,{ }^{* *} \mathrm{P}<0.01$, ICC test.

environment (37-39\%). Variability of the volume measures was to a similar degree determined by both familial and specific environmental factors independent of the hemisphere (Table 2).

Fornix. Familial effects significantly explained most of the variability in MD measure (73\%) of fornix. In contrast, familial effects had lower effect on the variability of FA and volume $(47 \%$ and $40 \%$, respectively) (Table 3 ).
Heritability of temporal-amygdala-orbitofrontal network. Uncinate fasciculus. High genetic effects significantly accounted for most of the variability in the left FA ( $80 \%)$ and right MD (58\%) of the uncinate fasciculus. It is important to note that the heritability of the right FA might be slightly inflated, because shared environmental factors might play a role as well in the $71 \%$ (ACE model was the best fit). Taken together, the familial effects explained $70-80 \%$ variability in the left, and $71-81 \%$ variability in the right 
hemisphere of MD and FA, respectively. Familial effects were also high for volume measures, accounting for $64 \%$ of the variance in the left, and $68 \%$ in the right hemisphere (Table 4).

\section{Discussion}

In this study, we have used DTI tractography to investigate heritability of different limbic tracts important for memory and integrated socio-emotional processing. To our knowledge, this is the first twin study in healthy adults based on multiple tractspecific measures of the limbic system pathways.

We observed the highest heritability for bilateral uncinate fasciculus, where genetic and/or familial factors explained 64$80 \%$ of variance in the microstructural measures of FA, MD and volume. This tract is the main connection of the temporoamygdala-orbitofrontal network that supports emotion processing, semantic cognition and social behaviour. Previous literature suggests an increase of the genetic effect on the FA of the uncinate fasciculus over time, with low heritability observed in neonates (Lee et al., 2015; but see Geng et al., 2012) and 9 year olds (Brouwer et al., 2010), but moderate heritability at the age of 12 (Brouwer et al., 2012). Our results suggest that genetic and familial effects might become increasingly important for uncinate fasciculus in adulthood. The findings are consistent with the recent voxel-wise report of high FA heritability in the uncinate fasciculus in adult population (Gatt et al., 2012). Many neuroimaging studies suggest an increase in heritability over time for specific brain measures, such as cortical thickness (Lenroot et al., 2009; van Soelen et al., 2012) or white matter volume (Wallace et al., 2006). The genetic effect on prosocial behaviour and empathy functions that this tract is likely to facilitate also increases with age (Ebstein et al., 2010). Several mechanisms might be responsible for this observed heritability change over time. One of these includes the active genotype-environment correlation, which reflects the increasing capacity to select environments that reinforce genetic dispositions. Second, childhood environments are largely determined by parents, but declining importance of shared environment across development could lead to a reduced environmental variance and an increase in heritability estimates (Bergen et al., 2007). Also, it is conceivable that measurement error decreases during this timeframe, because DTI in children is more prone to methodological and acquisition errors (motion artefacts, partial volume averaging, etc.) compared to adults. Reduced variance due to measurement error would manifest as an increase in heritability. Furthermore, the uncinate fasciculus has a prolonged maturation and its development continues well into third decade (Lebel et al., 2008). An increase in heritability may reflect the new set of genes being expressed during development, or an age-dependent gene expression, as genes turn 'on' and 'of' in response to developmental cues. Many highly heritable psychiatric disorders, which are associated with the uncinate fasciculus abnormalities, have their peak age of onset during adolescence (Paus et al., 2008), probably due to the age-dependent gene expression.

The high heritability of the uncinate fasciculus may have high significance for the understanding of certain neurodevelopmental conditions. We have previously reported, for example, that psychopathy is associated with reduced microstructural integrity of the uncinate fasciculus (Craig et al., 2009). The finding of a strong genetic influence on this tract is consistent with studies that show psychopathic personality (Viding et al., 2005; Blonigen et al., 2005), childhood aggression and adult crime to be highly heritable (Moffitt, 1993), and reported difficulties in ameliorating core emotional personality deficiencies associated with this disorder by psychotherapy (Lee 1999). Future longitudinal studies of the uncinate fasciculus are needed to assess the contribution of genes and environment over time, and their interaction with maturational processes.

Our results also seem to suggest differences in the heritability of dorsal (medial default-mode network) and ventral cingulum (hippocampal-diencephalic-retrosplenial network). The variability of the microstructure (FA, MD) of the left ventral cingulum is mostly explained by specific environmental effects (58\%, 68\%) compared to the right ventral cingulum, whose microstructural variability is predominantly explained by familial effects $(63 \%$, $61 \%$ ). Previously, differences in heritability estimates were related to differences in white matter asymmetries (Jahanshad et al., 2013; Budisavljevic et al., 2015), such that the tracts with higher FA exhibited higher heritability. The previously reported right lateralization of the ventral cingulum (Wakana et al., 2007) could explain the observed differences in heritability. However, our study found symmetrical distribution of FA in the ventral cingulum, consistent with other reports (Malykhin et al., 2008; Nakata et al., 2009), thus not supporting this hypothesis. The volume of the bilateral ventral cingulum was equally affected by familial and environmental factors. Contrary to the ventral cingulum, the microstructure of the dorsal cingulum was under high genetic and familial factors independent of the hemisphere. Genetic and familial factors accounted for $73 \%$ and $62 \%$ of the variance in the right and left MD, $54 \%$ and $58 \%$ in right and left FA, and $34 \%$ and $51 \%$ in the right and left volume of the dorsal cingulum. Our FA heritability estimates for the dorsal cingulum are much higher than those noted in neonates (15-23\%) (Lee et al., 2015), but are in line with the heritability reported in childhood (51-54\%; note however that these estimates are for the whole cingulum bundle) (Brouwer et al., 2012) and voxel-wise reports in adults (Kochunov et al., 2010; Gatt et al., 2012; Jahanshad et al., 2013). It is important to mention that FA of the cingulum has important functional relevance, and was found to be positively correlated with the full-scale and performance IQ with common genetic factors affecting both measures (Chiang et al., 2008, 2009). The only study that has also divided the cingulum into dorsal and ventral components in adult population did not investigate the same diffusion measures, and found small to moderate effects of genes on fibre orientation distribution (26-29\% for dorsal and 14-33\% for ventral cingulum) (Shen et al., 2014). However, they also found high heritability of connections to the cingulate gyri, which most likely involve the dorsal cingulum fibres. Consistent with our results, measures of default-mode network activity that dorsal cingulum connects are also found to be moderately to highly heritable (Castellanos et al., 2010; Glahn et al., 2010; Fornito et al., 2011). Our findings add further validity to the anatomical distinction between dorsal and ventral cingulum (Catani et al., 2013a,b; Jones et al., 2013), with high heritability estimates associated with the bilateral dorsal cingulum, implicated in social cognition and emotional processing (Bush et al., 2000; Amodio and Frith 2006), compared to the left ventral cingulum, more related to memory functions and spatial orientation (Damasio 1989; Heimer and Van Hoesen 2006).

Together with the ventral cingulum, fornix is part of the hippocampal-diencephalic-retrosplenial network important for memory (Metzler-Baddeley et al., 2011, 2012; Rolls, 2015). Fornix was under high familial effects for MD (68\%), and moderate for FA and volume (47\% and $46 \%)$. We did not have enough power to obtain significance for genetic and shared environmental effects individually. However, our ICC analysis suggests that genetics and shared environment might play a different role for FA and MD measures. Thus, additive genetic factors are likely to be more 
important for FA (MZ correlations more than double of DZ correlations), whereas shared environmental factors might affect MD more (MZ and DZ correlations equally high and significant). Previous studies on heritability of the fornix present conflicting results. DTI tractography studies show that fornix microstructure is highly heritable in neonates (43-62\%) (Lee et al., 2015), but not in children (18-21\%) (Brouwer et al., 2012). On the other hand, voxel-wise analyses in adults report both low (Jahanshad et al., 2013) and high heritability (Gatt et al., 2012) for the FA measure.

The implications of these findings may be significant to our understanding of heritability of social and emotional processing in terms of personality and healthy adult cognition, as well as disordered functioning. Nowadays, DTI measures are starting to be used as phenotypes for genome-wide association and linkage studies (Kochunov et al., 2011; Chiang et al., 2012; Jahanshad et al., 2012) for identifying novel variants and molecular pathways associated with white matter microstructure. In our study, genetic influence varied among different diffusion measures, in line with the previous findings in children (Brouwer et al., 2010) and adults (Budisavljevic et al., 2015). Although the specific microstructural correlates are yet to be fully elucidated, diffusion measures are differentially sensitive to the degree of myelination, axonal membrane integrity, axonal density and diameter (Beaulieu, 2002; Song et al., 2003; Budde et al., 2007). Thus, the differences in heritability might reflect differences in physiological mechanisms underlying each measure. In the future, multivariate approaches in larger samples are needed to decipher the shared genetic and environmental effects between these variables. Finally, differences in heritability might also reflect differences in the reliability of diffusion measures, which can be influenced by acquisition sequence and parameters. For example, the FA and MD are found to be more reproducible and reliable than volume (Heiervang et al., 2006; Wang et al., 2012). By exploring multiple DTI measures that capture different aspects of the limbic white matter anatomy, our study may empower the search for specific genetic polymorphisms that impact the limbic white matter structure. Similarly, reliability is also important when discussing the differences in heritability among different limbic tracts. Previously, the cingulum and the uncinate fasciculus were found to exhibit more reliable diffusion measures compared to the fornix (Wang et al., 2012). If this assumption is valid, the heritability estimates of the cingulum and uncinate would be inflated compared to the fornix, by reducing the variance error.

Although our study has a number of strengths it also has several limitations that should be taken into account. First, there are technical limitations of DTI tractography such as inability to solve crossing or kissing of fibres in a voxel, leading to the presence of false positives and false negatives (Dell'Acqua and Catani, 2012). However, all our limbic tracts were visually inspected to ensure that they conformed to known anatomical trajectories. It should be noted that noise in the tractography data and the ease of tracking may have increased the measurement error thus inflating the estimates of specific environment (E). Furthermore, standard limitations of the classical twin study design should be taken into account, such as assumptions of equal environments between MZ and DZ twins (Plomin and Kosslyn, 2001), problems with significant gene-environment correlations and interactions, lack of follow-up of the phenotypes over time, and environmental noise (Boomsma et al., 2002). The assumption of the equal environment across zygosities is especially important when investigating the social brain, because the nature of socialization was shown to affect myelination in animals (Liu et al., 2012) and the integrity of the uncinate fasciculus in humans (Eluvathingal et al., 2006).
Nevertheless, these effects were linked to severe socio-emotional deprivation, and the implications to the healthy (twin) population still remains to be established. Our study assumes similar degrees of social interaction between $\mathrm{MZ}$ and $\mathrm{DZ}$ twins. However, there is some evidence that MZ twins are treated more similarly by their parents and interact more frequently as adults than DZ twins, which could result in increased correlations for MZ Us DZ twins, and an overestimation of the genetic effects for phenotypes relevant to social cognition (Constantino and Todd, 2000). Nevertheless, the behavioural studies of twins reared apart argue in favour of the equal environment assumption, showing that the degree of social contact has no impact on behavioural similarity (Rijsdijk and Sham, 2002). Still, the heritability estimates from twin studies should be seen only as estimates, and should be interpreted carefully. The future studies should address the nature of social interaction in MZ Us DZ twins, and the effect it might have onto the limbic brain structures. Also, there is a question of whether the results of twin studies can be applicable to non-twin population, because twins are more likely than singletons to experience adverse prenatal and perinatal events that may affect brain development (Norwitz et al., 2005). Studies report that there are no significant differences in the brain structure between twins and singletons in adulthood (Hulshoff Pol et al., 2002; Knickmeyer et al., 2011). Nevertheless, our findings are valid only for the adult population, because heritability is known to change with age (Lenroot et al., 2009). For the standards of quantitative genetics, our twin study was further limited by sample size, leading to the confidence intervals of heritability estimates to be wide. Sample size impacts on model specification and this consideration dictated our choice of reporting estimates of the ACE model. A large sample is necessary to detect shared environmental (C) effects (Posthuma and Boomsma 2000). The reduction in power was overcome by reporting $C$ and $A$ (genetic) effects in combination, when they were statistically significant together, and expressing them as 'familial effects' (Wright et al., 2002). Finally, our study would have benefited from the inclusion of neuropsychological or behavioural measurements. This would have enabled us to complete a more comprehensive investigation of behavioural-neuroanatomical heritability.

In summary, our findings indicate a strong genetic and familial effects on the anatomy of the limbic pathways that underpin emotion processing, that is, uncinate fasciculus (temporo-amygdala-orbitofrontal network) and dorsal cingulum (medial default-mode network) and a weaker but still high effect on the tracts that underpin memory, that is, left ventral cingulum and fornix (hippocampal-diencephalic retrosplenial network). In the light of the differential role that these networks have with respect to social and cognitive functioning, these findings might have important translational implications.

\section{Acknowledgements}

We would like to thank the NatBrainLab (www.natbrainlab. com) for helpful comments on the manuscript.

\section{Funding}

This work was supported by the MRC UK (grant number G0400061) as the AIMS (Autism Imaging Multicentre Study) with support from the National Institute for Health Research (NIHR) Biomedical Research Centre at South London and Maudsley NHS Foundation Trust, King's College London, and the Sackler Institute for Translational Neurodevelopment. 
Additional funding was provided by the European Autism Interventions-A Multicentre Study for Developing New Medications (EU-AIMS), which received support from the Innovative Medicines Initiative Joint Undertaking (grant agreement number 115300), including financial contributions from the EU Seventh Framework Programme (FP7/2007-2013), the European Federation of Pharmaceutical Industries and Associations companies in kind, Autism Speaks, NARSAD, The Stanley Foundation, Schizophrenia Research Trust and Psychiatry Research Trust. The views expressed are those of the author and not necessarily those of the NHS, the NIHR or the Department of Health. M.C. is the recipient of a Welcome Trust Investigator Award (103759/Z/14/Z).

Conflict of interest. None declared.

\section{References}

Aggleton, J.P. (2008). EPS Mid-Career Award 2006. Understanding anterograde amnesia: disconnections and hidden lesions. Quarterly Journal of Experimental Psychology, 61(10), 1441-71.

Alhusaini, S., Whelan, C.D., Doherty, C.P., Delanty, N., Fitzsimons, M., Cavalleri, G.L. (2015). Temporal cortex morphology in mesial temporal lobe epilepsy patients and their asymptomatic siblings. Cerebral Cortex, pii: bhu315

Ameis, S.H., Catani, M. (2015). Altered white matter connectivity as a neural substrate for social impairment in Autism Spectrum Disorder. Cortex, 62, 158-81.

Amodio, D.M., Frith, C.D. (2006). Meeting of minds: the medial frontal cortex and social cognition. Nature Reviews Neuroscience, 7(4), 268-77.

Beaulieu, C. (2002). The basis of anisotropic water diffusion in the nervous system-a technical review. NMR in Biomedicine, 15(7-8), 435-55.

Bergen, S.E., Gardner, C.O., Kendler, K.S. (2007). Age-related changes in heritability of behavioural phenotypes over adolescence and young adulthood: a meta-analysis. Twin Research and Human Genetics, 10(3), 423-33.

Blonigen, D.M., Hicks, B.M., Krueger, R.F., Patrick, C.J., Iacono, W.G. (2005). Psychopathic personality traits: heritability and genetic overlap with internalizing and externalizing psychopathology. Psychological Medicine, 35, 637-48.

Boker, S., Neale, M., Maes, H., et al. (2011). OpenMx: an open source extended structural equation modeling framework. Psychometrika, 76(2), 306-17.

Boker, S.M., Neale, M.C., Maes, H.H., et al. (2012). OpenMx 1.3 User Guide. Charlottesville, VA: The OpenMx Project.

Boomsma, D., Busjahn, A., Peltonen, L. (2002). Classical twin studies and beyond. Nature Reviews Genetics, 3, 872-82.

Brouwer, R.M., Mandl, R.C.W., Peper, J.S., et al. (2010). Heritability of DTI and MTR in nine-year-old children. NeuroImage, 53(3), 1085-92.

Brouwer, R.M., Mandl, R.C.W., Schnack, H.G., et al. (2012). White matter development in early puberty: a longitudinal volumetric and diffusion tensor imaging twin study. PLoS One, 7(4), e32316.

Broyd, S.J., Demanuele, C., Debener, S., Helps, S.K., James, C.J., Sonuga-Barke, E.J.S. (2009). Default-mode brain dysfunction in mental disorders: a systematic review. Neuroscience and Biobehavioral Reviews, 33(3), 279-96.

Buckner, R.L., Snyder, A.Z., Shannon, B.J., et al. (2005). Molecular, structural, and functional characterization of Alzheimer's disease: evidence for a relationship between default activity, amyloid, and memory. Journal of Neuroscience, 25(34), 7709-17.

Budde MD., Kim JH., Liang HF., et al. (2007). Toward accurate diagnosis of white matter pathology using diffusion tensor imaging. Magnetic Resonance in Medicine, 57(4), 688-95.

Budisavljevic, S., Dell'Acqua, F., Rijsdijk, F.V., et al. Kane, F., Picchioni, M., McGuire, P., Toulopoulou, T., Georgiades, A., Kalidindi, S., Kravariti, E., Murray, R.M., Murphy, D.G., Craig, M.C., Catani, M. (2015) Age-related differences and heritability of the perisylvian language networks. Journal of Neuroscience, 35(37), 12625-34.

Bush, G., Luu, P., Posner, M.I. (2000). Cognitive and emotional influences in anterior cingulate cortex. Trends in Cognitive Sciences, 4(6), 215-22.

Carballedo, A., Amico, F., Ugwu, I., et al. (2012). Reduced fractional anisotropy in the uncinate fasciculus in patients with major depression carrying the met-allele of the Val66Met brain-derived neurotrophic factor genotype. American Journal of Medical Genetics. Part B, Neuropsychiatric Genetics, 159B(5), 537-48.

Castellanos, F.X., Zuo, X.N., Williams, K., et al., (2010). Genetic analyses of resting-state studies in adolescent twins: preliminary results. In: 16th Annual Meeting of the Organization of Human Brain Mapping, Barcelona, Spain, 6-10 June 2010.

Catani, M., Bambini, V. (2014). A model for social communication and language evolution and development (SCALED). Curr Opin Neurobiol, 28, 165-71.

Catani, M., Dell'acqua, F., Bizzi A, Forkel S.J., Williams, S.C., Simmons, A., Murphy, D.G., Thiebaut de Schotten, M. (2012). Beyond cortical localisation in clinico-anatomical correlation. Cortex, 48, 1262-87.

Catani, M., Dell'Acqua, F., Budisavljevic, S., et al. (in press). Frontal networks in adults with autism spectrum disorder. Brain.

Catani, M., Dell'Acqua, F., Thiebaut de Schotten, M. (2013a). A revised limbic system model for memory, emotion and behaviour. Neuroscience and Biobehavioral Reviews, 37(8), 1724-37.

Catani, M., Howard, R.J., Pajevic, S., Jones, D.K. (2002). Virtual in vivo interactive dissection of white matter fasciculi in the human brain. NeuroImage, 17(1), 77-94.

Catani M., Mesulam, MM. (2008). The arcuate fasciculus and the disconnection theme in language and aphasia: history and current state. Cortex, 44, 953-61.

Catani, M., Mesulam, M.M., Jakobsen, E., et al. (2013b). A novel frontal pathway underlies verbal fluency in primary progressive aphasia. Brain, 136(Pt 8), 2619-28.

Catani M., Thiebaut de Schotten, M. (2008). A diffusion tensor imaging tractography atlas for in vivo dissections. Cortex, 44 , 1105-32.

Catheline, G., Periot, O., Amirault, M., Braun, M., Dartigues, J.F., Auriacombe, S., Allard, M. (2010). Distinctive alterations of the cingulum bundle during aging and Alzheimer's disease. Neurobiology of Aging, 31(9), 1582-92.

Chiang, M.C., Barysheva, M., Lee, A.D., et al. (2008). Brain fiber architecture, genetics and intelligence: a high angular resolution diffusion imaging (HARDI) study. Medical Image Computing and Computer-Assisted Intervention, 11(Pt 1), 1060-7.

Chiang, M.C., Barysheva, M., McMahon, K.L., et al. (2012). Gene network effects on brain microstructure and intellectual performance identified in 472 twins. Journal of Neuroscience, 32(25), 8732-45.

Chiang, M.C., Barysheva, M., Shattuck, D.W., et al. (2009). Genetics of brain fiber architecture and intellectual performance. Journal of Neuroscience, 29(7), 2212-24. 
Concha, L., Livy, D.J., Beaulieu, C., Wheatley, B.M., Gross, D.W. (2010). In vivo diffusion tensor imaging and histopathology of the fimbria-fornix in temporal lobe epilepsy. Journal of Neuroscience, 30(3), 996-1002.

Constantino, J.N., Todd, R.D. (2000). Genetic structure of reciprocal social behavior. American Journal of Psychiatry, 157, 2043-5.

Craig, I.W., Halton, K.E. (2009). Genetics of human aggressive behaviour. Human Genetics, 126, 101-13.

Craig, M.C., Catani, M., Deeley, Q., et al. (2009). Altered connections on the road to psychopathy. Molecular Psychiatry, 14(10), 946-53.

Damasio, A.R. (1989). Time-locked multiregional retroactivation: a systems-level proposal for the neural substrates of recall and recognition, Cognition, 33(1-2), 25-62.

D’Anna, L., Mesulam, M.M., Thiebaut de Schotten, M., et al. (2015). Abnormalities of the uncinate fasciculus correlate with behavioral symptoms in primary progressive aphasia. Neurology.

Dell'Acqua, F., Catani, M. (2012). Structural human brain networks: hot topics in diffusion tractography. Current Opinion in Neurology, 25(4), 375-83.

Douet, V., Chang, L. (2014). Fornix as an imaging marker for episodic memory deficits in healthy aging and in various neurological disorders. Frontiers in Aging Neuroscience, 6, 343.

Eaves, L., Heath, A., Martin, N., et al. (1999). Comparing the biological and cultural inheritance of personality and social attitudes in the Virginia 30,000 study of twins and their relatives. Twin Research, 2(2), 62-80.

Ebstein, R.P., Israel, S., Chew, S.H., Zhong, S., Knafo, A. (2010). Genetics of human social behavior. Neuron, 65(6), 831-44.

Elison, J.T., Wolff, J.J., Heimer, D.C., et al. (2013). Frontolimbic neural circuitry at 6 months predicts individual differences in joint attention at 9 months. Developmental Science, 16(2), 18697.

Eluvathingal, T.J., Chugani, H.T., Behen, M.E., et al. (2006). Abnormal brain connectivity in children after early severe socioemotional deprivation: a diffusion tensor imaging study. Pediatrics, 117(6), 2093-100.

Fitzsimmons, J., Schneiderman, J.S., Whitford, T.J., et al. (2014). Cingulum bundle diffusivity and delusions of reference in first episode and chronic schizophrenia. Psychiatry Research, 224(2), 124-32.

Fornito, A., Zalesky, A., Bassett, D.S., et al. (2011). Genetic influences on cost-efficient organization of human cortical functional networks. Journal of Neuroscience, 31(9), 3261-70.

Fujie, S., Namiki, C., Nishi, H., et al. (2008). The role of the uncinate fasciculus in memory and emotional recognition in amnestic mild cognitive impairment. Dementia and Geriatric Cognitive Disorders, 26(5), 432-9.

Gatt, J.M., Korgaonkar, M.S., Schofield, P.R., et al. (2012). The TWIN-E project in emotional wellbeing: study protocol and preliminary heritability results across four MRI and DTI measures. Twin Research and Human Genetics, 15(3), 419-41.

Geng, X., Prom-Wormley, E.C., Perez, J., et al. (2012) White matter heritability using diffusion tensor imaging in neonatal brains. Twin Research and Human Genetics, 15(3), 336-50.

Glahn, D.C., Winkler, A.M., Kochunov, P., et al. (2010). Genetic control over the resting brain. Proceedings of the National Academy of Sciences of the United States of America, 107(3), 1223-8.

Heiervang, E., Behrens, T.E.J., Mackay, C.E., Robson, M.D., Johansen-Berg, H. (2006). Between session reproducibility and between subject variability of diffusion MR and tractography measures. NeuroImage, 33(3), 867-77.

Heimer, L., Van Hoesen, G.W. (2006). The limbic lobe and its output channels: implications for emotional functions and adaptive behavior. Neuroscience and Biobehavioral Reviews, 30(2), 126-47.

Hollocks, M.J., Lawrence, A.J., Brookes, R.L., et al. (2015). Differential relationships between apathy and depression with white matter microstructural changes and functional outcomes. Brain, 138, 3803-15.

Hulshoff Pol, H.E., Posthuma, D., Baaré, W.F.C., et al. (2002). Twinsingleton differences in brain structure using structural equation modelling. Brain, 125(Pt 2), 384-90.

Hulshoff Pol, H.E., Schnack, H.G., Posthuma, D., et al. (2006). Genetic contributions to human brain morphology and intelligence. Journal of Neuroscience, 26(40), 10235-42.

Jahanshad, N., Kochunov, P.V., Sprooten, E., et al. (2013). Multisite genetic analysis of diffusion images and voxelwise heritability analysis: a pilot project of the ENIGMA-DTI working group. NeuroImage, 81, 455-69.

Jahanshad, N., Kohannim, O., Toga, A.W., McMahon, K.L., de Zubicaray, G.I., Hansell, N.K., et al. (2012). Diffusion imaging protocol effects on genetic associations. Proc IEEE Int Symp Biomed Imaging. 944-7.

Järvenpää, T., Laakso, M.P., Rossi, R., et al. (2004). Hippocampal MRI volumetry in cognitively discordant monozygotic twin pairs. Journal of Neurology, Neurosurgery, and Psychiatry, 75(1), 116-20.

Jones, D.K., Christiansen, K.F., Chapman, R.J., Aggleton, J.P. (2013). Distinct subdivisions of the cingulum bundle revealed by diffusion MRI fibre tracking: implications for neuropsychological investigations. Neuropsychologia, 51(1), 67-78.

Kamagata, K., Motoi, Y., Abe, O., Shimoji, K., Hori, M., Nakanishi, A., et al. (2012). White matter alteration of the cingulum in Parkison Disease with and without dementia: Evaluation by diffusion tensor tract-specific analysis. AJNR Am J Neuroradiology, 33(5), 890-5.

Kim, S.J., Jeong, D.U., Sim, M.E., Bae, S.C., Chung, A., Kim M.J., et al. (2006). Asymmetrically altered integrity of cingulum bundle in posttraumatic stress disorder. Neuropsychobiology, 54(2), $120-5$.

Knafo, A., Israel, S. (2009). Genetic and environmental influences on prosocial behavior. In: Mikulincer, M., Shaver, P.R., editors. Prosocial Motives, Emotions, and Behavior: The Better Angels of Our Nature, M. Mikulincer and P.R. Shaver, eds. (Washington, DC: APA Books).

Knickmeyer, R.C., Kang, C., Woolson, S., et al. (2011). Twin-singleton differences in neonatal brain structure. Twin Research and Human Genetics, 14(3), 268-76.

Koch, K., Schultz, C.C., Wagner, G., et al. (2013). Disrupted white matter connectivity is associated with reduced cortical thickness in the cingulate cortex in schizophrenia. Cortex, 49(3), 722-9.

Kochunov, P., Glahn, D.C., Nichols, T.E., Winkler, A.M., Hong, E.L., Holcomb, H.H., et al. (2011). Genetic analysis of cortical thickness and fractional anisotropy of water diffusion in the brain. Frontiers in Neuroscience, 5, 120.

Kochunov, P., Glahn, D.C., Lancaster, J.L., et al. (2010). Genetics of microstructure of cerebral white matter using diffusion tensor imaging. NeuroImage, 53(3), 1109-16.

Kremen, W.S., Prom-Wormley, E., Panizzon, M.S., et al. (2010). Genetic and environmental influences on the size of specific brain regions in midlife: the VETSA MRI study. NeuroImage, 49(2), 1213-23.

Kubicki, M., Westin, C.F., Nestor, P.G., et al. (2003). Cingulate fasciculus integrity disruption in schizophrenia: a magnetic resonance diffusion tensor imaging study. Biological Psychiatry, 54(11), 1171-80. 
Kumar, A., Sundaram, S.K., Sivaswamy, L., et al. (2010). Alterations in frontal lobe tracts and corpus callosum in young children with autism spectrum disorder. Cerebral Cortex, 20(9), 2103-13.

Lebel, C., Walker, L., Leemans, A., Phillips, L., Beaulieu, C. (2008). Microstructural maturation of the human brain from childhood to adulthood. NeuroImage, 40(3), 1044-55.

Lee, J.H. (1999). The treatment of psychopathic and antisocial personality disorders: a review. In: Clinical Decision Making Support Unit, Broadmoor Hospital Berkshire, 1-33.

Lee, S.J., Steiner, R.J., Luo, S., et al. (2015). Quantitative tract-based white matter heritability in twin neonates. NeuroImage, 111, 123-35.

Leemans, A., Jones, D.K. (2009). The B-matrix must be rotated when correcting for subject motion in DTI data. Magnetic Resonance in Medicine, 61(6), 1336-49.

Lenroot, R.K., Schmitt, J.E., Ordaz, S.J., et al. (2009). Differences in genetic and environmental influences on the human cerebral cortex associated with development during childhood and adolescence. Human Brain Mapping, 30(1), 163-74.

Lipsman, N., Woodside, D.B., Lozano, A.M. (2015). Neurocircuitry of limbic dysfunction in anorexia nervosa. Cortex, 62, 109-18.

Lischke, A., Domin, M., Freyberger, H.J., et al. (2015). Structural alterations in white-matter tracts connecting (para)-limbic and prefrontal brain regions in borderline personality disorder. Psychological Medicine, 45(15), 3171-80.

Liu, J., Dietz, K., DeLoyht, J.M., et al. (2012). Impaired adult myelination in the prefrontal cortex of socially isolated mice. Nature Neuroscience, 15(12), 1621-3.

Losoya, S.H., Callor, S., Rowe, D.C., Goldsmith, H.H. (1997). Origins of familial similarity in parenting: a study of twins and adoptive siblings. Developmental Psychology, 33, 1012-23.

Malykhin, N., Concha, L., Seres, P., Beaulieu, C., Coupland, N.J. (2008). Diffusion tensor imaging tractography and reliability analysis for limbic and paralimbic white matter tracts. Psychiatry Research, 164(2), 132-42.

Matthews, K.A., Batson, C.D., Horn, J., Rosenman, R.H. (1981). "Principles in his nature which interest him in the fortune of others ...": the heritability of empathic concern for others. Journal of Personality, 49(3), 237-47.

McDonald, C., Bullmore, E.T., Sham, P.C., et al. (2004). Association of genetic risks for schizophrenia and bipolar disorder with specific and generic brain structural endophenotypes. Archives of General Psychiatry, 61(10), 974-84.

McIntosh, A.M., Moorhead, T.W.J., Job, D., et al. (2008). The effects of a neuregulin 1 variant on white matter density and integrity. Molecular Psychiatry, 13(11), 1054-9.

Mesulam, M. (2000). Brain, mind, and the evolution of connectivity. Brain and Cognition, 42(1), 4-6.

Metzler-Baddeley, C., Hunt, S., Jones, D.K., Leemans, A., Aggleton, J.P., O’Sullivan, M.J. (2012). Temporal association tracts and the breakdown of episodic memory in mild cognitive impairment. Neurology, 79(23), 2233-40.

Metzler-Baddeley, C., Jones, D.K., Belaroussi, B., Aggleton, J.P., O'Sullivan, M.J. (2011). Frontotemporal connections in episodic memory and aging: a diffusion MRI tractography study. The Journal of Neuroscience, 31(37), 13236-45.

Moffitt, T.E. (1993). Adolescence-limited and life-coursepersistent antisocial behavior: a developmental taxonomy. Psychological Review, 100(4), 674-701.

Mori, T., Ohnishi, T., Hashimoto, R., et al. (2007). Progressive changes of white matter integrity in schizophrenia revealed by diffusion tensor imaging. Psychiatry Research, 154(2), 13345.
Nakata Y., Barkovich AJ., Wahl M., et al. (2009). Diffusion abnormalities and reduced volume of the ventral cingulum bundle in agenesis of the corpus callosum: a 3T imaging study. American Journal of Neuroradioly, 30(6), 1142-8.

Neale, M.C., Maes, H.H. (2002). Methodology for Genetic Studies of Twins and Families. Dordecht: Kluwer.

Neale, M.C., Eaves, L.J., Kendler, K.S. (1994). The power of the classical twin study to resolve variation in threshold traits. Behavior Genetics, 24(3), 239-58.

Nestor, P.J., Fryer, T.D., Smielewski, P., Hodges, J.R. (2003). Limbic hypometabolism in Alzheimer's disease and mild cognitive impairment. Annals of Neurology, 54(3), 343-51.

Nortje, G., Stein, D.J., Radua, J., Mataix-Cols, D., Horn, N. (2013). Systematic review and voxel-based meta-analysis of diffusion tensor imaging studies in bipolar disorder. Journal of Affective Disorders, 150(2), 192-200.

Norwitz, E.R., Edusa, V., Park, J.S. (2005). Maternal physiology and complications of multiple pregnancy. Seminars in Perinatology, 29(5), 338-48.

Paus, T., Keshavan, M., Giedd, J.N. (2008). Why do many psychiatric disorders emerge during adolescence?. Nature Reviews Neuroscience, 9(12), 947-57.

Peng, Z., Shi, F., Shi, C., et al. (2014). Structural and diffusion property alterations in unaffected siblings of patients with obsessive-compulsive disorder. PLoS One, 9(1), e85663.

Plomin, R., Kosslyn, S.M. (2001). Genes, brain and cognition. Nature Neuroscience, 4(12), 1153-4.

Plomin, R., DeFries, J.C., Knopik, V.S., Neiderheiser, J. (2013). Behavioral Genetics. New York: Palgrave Macmillan.

Plomin, R., Owen, M.J., McGuffin, P. (1994). The genetic basis of complex human behaviors. Science, 264(5166), 1733-9.

Posthuma, D., Boomsma, D.I. (2000). A note on the statistical power in extended twin designs. Behaviour Genetics, 30(2), 14758.

Pugliese, L., Catani, M., Ameis, S., et al. (2009). The anatomy of extended limbic pathways in Asperger syndrome: a preliminary diffusion tensor imaging tractography study. NeuroImage, 47(2), 427-34.

Rijsdijk, F.V., Sham, P.C. (2002). Analytic approaches to twin data using structural equation models. Briefings in Bioinformatics, 3(2), 119-33.

Rijsdijk, F.V., van Haren, N.E.M., Picchioni, M.M., et al. (2005). Brain MRI abnormalities in schizophrenia: same genes or same environment? Psychological Medicine, 35(10), 1399-409.

Roalf, D.R., Vandekar, S.N., Almasy, L., et al. (2015). Heritability of subcortical and limbic brain volume and shape in multiplexmultigenerational families with schizophrenia. Biological Psychiatry, 77(2), 137-46.

Rolls, E.T. (2015). Limbic systems for emotions and for memory, but no single limbic system. Cortex, 62, 119-57.

Rushton, J.P., Fulker, D.W., Neale, M.C., Blizard, R.A., Eysenck, H.J. (1984). Altruism and genetics. Acta Geneticae Medicae et Gemellologiae, 33(2), 265-71.

Rushton, J.P., Fulker, D.W., Neale, M.C., Nias, D.K., Eysenck, H.J. (1986). Altruism and aggression: the heritability of individual differences. Journal of Personality and Social Psychology, 50(6), 1192-8.

Scanlon, C., Ronan, L., Doherty, C.P., et al. (2013). MRI-based brain structure volumes in temporal lobe epilepsy patients and their unaffected siblings: a preliminary study. Journal of Neuroimaging, 23(1), 64-70.

Schulte, T., Müller-Oehring, E.M., Sullivan, E.V., Pfefferbaum, A. (2012). White matter fiber compromise contributes differentially to attention and emotion processing impairment in 
alcoholism, HIV-infection, and their comorbidity. Neuropsychologia, 50(12), 2812-22.

Scourfield, J., Martin, N., Lewis, G., McGuffin, P. (1999). Heritability of social cognitive skills in children and adolescence. British Journal of Psychiatry, 175, 559-64.

Sethi, A., Gregory, S., Dell'Acqua, F., et al. (2015). Emotional detachment in psychopathy: involvement of dorsal defaultmode connections. Cortex, 62, 11-9.

Sheline, Y.I., Barch, D.M., Price, J.L., et al. (2009). The default mode network and self-referential processes in depression. Proceedings of the National Academy of the Sciences of the United States of America, 106(6), 1942-7.

Shen, K.K., Rose, S., Fripp, J., et al. (2014). Investigating brain connectivity heritability in a twin study using diffusion imaging data. NeuroImage, 100, 628-41.

Song, S.K., Sun, S.W., Ju, W.K., Lin, S.J., Cross, A.H., Neufeld, A.H. (2003). Diffusion tensor imaging detects and differentiates axon and myelin degeneration in mouse optic nerve after retinal ischemia. NeuroImage, 20(3), 1714-22.

Stein, M.B., Jang, K.L., Livesley, W.J. (2002). Heritability of social anxiety-related concerns and personality characteristics: a twin study. Journal of Nervous and Mental Disease, 190(4), 219-24.

Stromswold, K. (2001). The heritability of language: a review and metaanalysis of twin, adoption, and linkage studies. Language, 77, 647-723.

Sun, Z., Wang, F., Cui, L., Breeze, J., Du, X., Wang, X., et al. (2003). Abnormal anterior cingulum in patients with schizophrenia: a diffusion tensor imaging study. Neuroreport, 14(14), 1833-6.

Takei, K., Yamasue, H., Abe, O., Yamada, H., Inoue, H., Suga, M., et al. (2009). Structural disruption of the dorsal cingulum bundle is associated with impaired Stroop performance in patients with schizophrenia. Schizophrenia Research, 114(1-3), 119-27.

Thapar, A., Petrill, S.A., Thompson, L.A. (1994). The heritability of memory in the Western Reserve Twin Project. Behavioral Genetics, 24(2), 155-60.

Thiebaut de Schotten, M., ffytche, D.H., Bizzi, A., Dell'Acqua, F., Allin, M., Walshe, M., Murray, R., Williams, S.C., Murphy, D.G., Catani, M. (2011). Atlasing location, asymmetry and inter-subject variability of white matter tracts in the human brain with MR diffusion tractography. Neuroimage, 54, 49-59.

Thomas, C., Humphreys, K., Jung, K.J., Minshew, N., Behrmann, M. (2011). The anatomy of the callosal and visual-association pathways in high-functioning autism: a DTI tractography study. Cortex, 47(7), 863-73.

Valenstein, E., Bowers, D., Verfaellie, M., Heilman, K.M., Day, A., Watson, R.T. (1987). Retrosplenial amnesia. Brain, 110(Pt 6), 1631-46.

van Haren, N.E.M., Rijsdijk, F., Schnack, H.G., et al. (2012). The genetic and environmental determinants of the association between brain abnormalities and schizophrenia: the schizophrenia twins and relatives consortium. Biological Psychiatry, 71(10), 915-21.

van Soelen, I.L., Brouwer, R.M., Peper, J.S., et al. (2012). Brain SCALE: brain structure and cognition: an adolescent longitudinal twin study into the genetic etiology of individual differences. Twin Research and Human Genetics, 15(3), 453-67.

Vann, S.D., Aggleton, J.P., Maguire, E.A. (2009). What does the retrosplenial cortex do?. Nature Reviews Neuroscience, 10(11), 792-802.

Viding, E., Blair, R.J., Moffitt, T.E., Plomin, R. (2005). Evidence for substantial genetic risk for psychopathy in 7-year-olds. Journal of Child Psychology and Psychiatry, 46(6), 592-7.

Wakana, S., Caprihan, A., Panzenboeck, M.M., et al. (2007). Reproducibility of quantitative tractography methods applied to cerebral white matter. NeuroImage, 36(3), 630-44.

Wallace GL., Eric Schmitt J., Lenroot R., et al. (2006). A pediatric twin study of brain morphometry. Journal of Child Psychology and Psychiatry, 47(10), 987-93.

Wang, J.Y., Abdi, H., Bakhadirov, K., Diaz-Arrastia, R., Devous, M.D., Sr. (2012). A comprehensive reliability assessment of quantitative diffusion tensor tractography. NeuroImage, 60(2), 1127-38.

Weinberger, D.R., Berman, K.F., Suddath, R., Torrey, E.F. (1992). Evidence of dysfunction of a prefrontal-limbic network in schizophrenia: a magnetic resonance imaging and regional cerebral blood flow study of discordant monozygotic twins. The American Journal of Psychiatry, 149(7), 890-7.

Whitford, T.J., Kubicki, M., Pelavin, P.E., et al. (2015). Cingulum bundle integrity associated with delusions of control in schizophrenia: preliminary evidence from diffusion-tensor tractography. Schizophrenia Research, 161(1), 36-41.

Wright, I.C., Sham, P., Murray, R.M., Weinberger, D.R., Bullmore, E.T. (2002). Genetic contributions to regional variability in human brain structure: methods and preliminary results. NeuroImage, 17(1), 256-71.

Wu, T.C., Wilde, E.A., Bigler, E.D., Yallampalli, R., McCauley, S.R., Troyanskaya, M., et al. (2010). Evaluating the relationship between memory functioning and cingulum bundles in acute mild traumatic brain injury using diffusion tensor imaging. Journal of Neurotrauma, 27(2), 303-7.

Zhang, A., Leow, A., Ajilore, O., et al. (2012). Quantitative tract-specific measures of uncinate and cingulum in major depression using diffusion tensor imaging. Neuropsychopharmacology, 37, 959-67.

Zhang, Y., Schuff, N., Jahng, G.H., Bayne, W., Mori, S., Schad, L., et al. (2007). Diffusion tensor imaging of cingulum fibers in mild cognitive impairment and Alzheimer disease. Neurology, 68(1), 13-19. 